\title{
A STABLE/UNSTABLE "MANIFOLD” THEOREM FOR AREA PRESERVING HOMEOMORPHISMS OF TWO MANIFOLDS
}

\author{
STEWART BALDWIN AND EDWARD E. SLAMINKA
}

(Communicated by Kenneth R. Meyer)

\begin{abstract}
The stable/unstable manifold theorem for hyperbolic diffeomorphisms has proven to be of extreme importance in differentiable dynamics. We prove a stable/unstable "manifold" theorem for area preserving homeomorphisms of orientable two manifolds having isolated fixed points of index less than 1. The proof relies upon the concept of free modification which was first developed by Morton Brown for homeomorphisms of two manifolds and later extended by Pelikan and Slaminka for area preserving homeomorphisms of two manifolds.
\end{abstract}

\section{INTRODUCTION}

The stable/unstable manifold theorem (also known as the Hadamard-Perron theorem) for hyperbolic fixed points is a cornerstone in differentiable dynamics. The proof of this result has even filtered down to an advanced undergraduate level textbook in dynamics [De]. A topological version of this theorem, however, is still wanting. In the case of expansive homeomorphisms, both Lewowicz [Le] and Hiraide [Hi] have shown the existence of stable/unstable sets. These were needed to prove that there do not exist expansive homeomorphisms of the two spheres.

At least since Poincaré, there has been much attention given to area preserving homeomorphisms and symplectic diffeomorphisms such as twist maps of the annulus or, more recently, the Arnol'd conjecture [cf. Ar, Appendix 9]. Montgomery [Mo] showed that if $h: \mathbf{R}^{n} \rightarrow \mathbf{R}^{n}$ is a measure-preserving homeomorphism, then given any open neighborhood $U$ of a fixed point $p$ of $h$ there exists a compact connected set $K$ of which $p$ is a proper subset and such that $h(K) \subset K$. Ding [Di], in work concerning Duffing's equation, proved the existence of Lagrange stable sets for area-preserving homeomorphisms of the plane.

Received by the editors December 18, 1988 and, in revised form, May 8, 1989.

1980 Mathematics Subject Classification (1985 Revision). Primary 58F05.

Key words and phrases. Fixed point, index, stable and unstable manifold, area preserving. 
In this paper we present a topological analog of the stable/unstable manifold theorem for area-preserving, orientation-preserving homeomorphisms of orientable two manifolds.

We first prove the local version of the theorem in which we construct compact, connected, invariant sets which have area zero and which do not separate the plane, and then extend the stable (respectively, unstable) sets by applying $h^{-1}$ (respectively, $h$ ). The main tool which is used in this paper is that of free modification of an area-preserving homeomorphism. M. Brown $\left[\mathrm{Br}_{1}\right]$ originated this method for homeomorphisms in order to study index-related problems on two manifolds. Pelikan-Slaminka [PS] and Slaminka [Sl] have extended these techniques to area-preserving homeomorphisms of two manifolds. Though we define free modifications below and describe their properties, we refrain from reiterating the arguments which have been used in [PS] and [Sl] in order to keep this work brief.

In $\S 1$ we present the definitions and lemmas needed in the main theorem. In $\S 2$ we prove the main result.

We wish to thank the referee for his/her insightful comments and suggestions.

\section{Preliminary definitions AND lemmas}

We first define the notion of index of a simple closed curve.

Definition. Let $h: \mathbf{R}^{2} \rightarrow \mathbf{R}^{2}$ be a homeomorphism of the plane and let $C$ be a simple closed curve with $C \cap \operatorname{Fix}(h)=\varnothing$. The index of $C$ with respect to $h$, denoted $\operatorname{ind}(h, C)$, is the degree of the mapping

$$
H(x)=\frac{h(x)-x}{\|h(x)-x\|}
$$

restricted to $C$. The index of an isolated fixed point $p$ is inherited from a simple closed curve which bounds a disc $E$ such that $p \in \operatorname{int}(E)$ and $E \cap$ $F \operatorname{Fix}(h)=p$.

Definition. [ $\left.\mathrm{Br}_{1}\right]$ Let $h: M \rightarrow M$ be an orientation-preserving homeomorphism of an orientable two manifold $M$, and $E$ a topological disc in $M$ such that $h(E) \cap E=\varnothing$. A free modification $h g$ of $h$ is the composition of $h$ with a homeomorphism $g: M \rightarrow M$ which is supported on $E$ (i.e. $g(x)=x$ for all $x \in M-E)$.

A free modification of a homeomorphism $h$ does not alter the fixed point set nor the index of any simple closed curve.

The Brouwer lemma is essential in working with homeomorphisms which are devoid of index one simple closed curves.

Brouwer lemma. Let $h: \mathbf{R}^{2} \rightarrow \mathbf{R}^{2}$ be an orientation-preserving homeomorphism of the plane and let $E$ be a disc in $\mathbf{R}^{2}$ such that $E \cap h(E)=\varnothing$. If $E \cap h^{n}(E) \neq \varnothing$ for some $n>1$, then there exists a simple closed curve $C$ with $\operatorname{ind}(h, C)=1$.

For a proof of this lemma the reader should consult either Brown $\left[\mathrm{Br}_{1}\right]$ or Fathi [Fa]. 
The following corollary provides the first link with area-preserving homeomorphisms.

Corollary. Let $h: \mathbf{R}^{2} \rightarrow \mathbf{R}^{2}$ be an area-preserving, orientation-preserving homeomorphism of the plane and let $K \subset \mathbf{R}^{2}$ be a disc. If there exists a disc $E \subset K$ such that $h^{n}(E) \subset K$ for all $n>0$ and $E \cap \operatorname{Fix}(h)=\varnothing$, then there exists a simple closed curve $C \subset K$ having index one.

An easy consequence of the properties of index is that if $\operatorname{Fix}(h)$ is isolated and $\operatorname{ind}(h, p)<1$ for all $p \in \operatorname{Fix}(h)$, then there do not exist any simple closed curves of index one. The following lemma by Brown $\left[\mathrm{Br}_{2}\right]$ describes the $\omega$-limit set of a point for such homeomorphisms.

Brown lemma. Let $h: \mathbf{R}^{2} \rightarrow \mathbf{R}^{2}$ be an orientation preserving homeomorphism of the plane having no simple closed curves of index one. If the positive orbit of a point $x$ is bounded, then $\lim _{n \rightarrow \infty} f^{n}(x) \in \operatorname{Fix}(h)$.

\section{MAIN THEOREM}

Theorem. Let $h: M \rightarrow M$ be an area-preserving, orientation-preserving homeomorphism of an orientable two manifold $M$ having an isolated fixed point $p$ of index $-n$ for $n \geq 0$. Then there exists a topological disc $D$ with $p \in \operatorname{int}(D)$, $\operatorname{Fix}(h) \cap D=p$, having boundary $C$ with Lebesgue measure 0 , and there exist $2(n+1)$ compact, connected, area zero sets $S_{0}, S_{1}, \ldots, S_{n}, U_{0}, U_{1}, \ldots, U_{n}$ which do not separate $M$ such that:

(1) $U_{i}, S_{i} \subset D$ and $U_{i}, S_{i}$ meet $b d(D)$ for all $i$,

(2) $p \in U_{i}, S_{i}$ for all $i$,

(3) $h\left(S_{i}\right) \subset S_{i}, h^{-1}\left(U_{i}\right) \subset U_{i}$ for all $i$,

(4) if $x \in S_{i}$, then $h^{m}(x) \rightarrow p$ as $m \rightarrow \infty$, and similarly if $x \in U_{i}$, then $h^{-m}(x) \rightarrow p$ as $m \rightarrow \infty$; and,

(5) $S_{i} \cap S_{j}=p=U_{i} \cap U_{j}$ for all $i \neq j$.

(6) The sets $\left(S_{i} \cap C\right)$ and $\left(U_{i} \cap C\right)$ alternate on $C$.

Proof.

First reduction step. Since this result is purely local in nature we can reduce the problem, either by using the covering space of $M^{2}-\operatorname{Fix}(h) \cup\{p\}$ or by using the manifold charts, to the following setting. The manifold $M$ is $\mathbf{R}^{2}$, $h$ is an orientation-preserving, area-preserving homeomorphism of $\mathbf{R}^{2}$ and Fix $(h)$ consists of a countable collection of isolated fixed points $p_{i}$ such that $\operatorname{ind}\left(h, p_{i}\right)=-n$. We single out any one of these fixed points, designating it by $p$ and let $D_{1}$ be a disc with $p \in \operatorname{int}\left(D_{1}\right)$ and $D_{1} \cap \operatorname{Fix}(h)=p$.

Second reduction step. Using the method of free modifications for area-preserving homeomorphisms we can construct a simple closed curve $C$ with $h(C) \cup$ $h^{-1}(C) \subset \operatorname{int}\left(D_{1}\right)$ and $\operatorname{area}(C)=0$ which bounds a disc $D$ having $p \in \operatorname{int}(D)$, 
and area-preserving homeomorphism $\hat{h}$, such that $C$ is in the following canonical form:

(1) $C \cap \hat{h}(C)$ is finite,

(2) $C$ intersects $\hat{h}(C)$ transversely; and,

(3) $D \cap \hat{h}(D)$ is connected

(where by transverse intersection we mean that there exists a homeomorphism of a neighborhood of a point $x \in C \cap \hat{h}(C)$ to $\mathbf{R}^{2}$ such that $x$ is mapped to the origin and an arc on $C$ containing $x$ is mapped to an interval on the $x$-axis and an arc on $\hat{h}(C)$ containing $x$ is mapped to an interval on the $y$-axis). Thus such a simple closed curve $C$ and its image $\hat{h}(C)$ bounding discs $D$ and $\hat{h}(D)$ with a point $p \in \operatorname{int}(D \cap \hat{h}(D))$ of index $-n$ will have the form shown in Figure 1 (where, for example, $n=2$ ). Since the details explaining this reduction are given in full in [SI] they will not be repeated here. However, the salient features of this construction will be listed below. Let $a_{i}, b_{i}$ denote the endpoints on the arcs $\overline{(C-\hat{h}(D))}$ for $i=0,1, \ldots, n$. Let $\left\{\alpha_{i}\right\} \quad i=0, \ldots n$, be the disjoint arcs on $C-\hat{h}(D)$ from $b_{i}$ to $a_{i}$ where $\alpha_{n}$ is the arc on $C$ from $b_{n}$ to $a_{0}$. The necessary properties of this reduction by free modification are:

(1) $\hat{h}=h$ on $\mathbf{R}^{2}-D_{1}$,

(2) $\hat{h}=h$ on a small connected neighborhood $N(p) \subset \operatorname{int}\left(D_{1}\right)$ with $p \in$ $N(p)$,

(3) $\hat{h}$ is isotopic to $h$ on $D_{1}-N(p)$ by an isotopy which is fixed-point-free and equal to $h$ on $b d\left(D_{1}\right) \cup b d(N(p))$,

(4) the number of points of $C \cap \hat{h}(C)$ is $2(n+1)$,

(5) $\hat{h}$ has no simple closed curves of index one.

(6) If $x \in D$ and $\hat{h}^{N}(x) \notin D$ for some positive integer $N$, then $\hat{h}^{m}(x) \notin$ $D$ for all $m \geq N$.

The Brouwer lemma plus (1), (2), and (3) imply that $\operatorname{Fix}(\hat{h})=\operatorname{Fix}(h)$ and $\operatorname{ind}(\hat{h}, q)=\operatorname{ind}(h, q)$ for all $q \in \operatorname{Fix}(h)$.

Construction of the invariant sets. Let $\left\{A_{i}\right\}, i=0, \ldots, n$ denote the discs $\hat{h}(D)-D$ bounded by the arcs $\delta_{i}, \beta_{i}$ where the endpoints of $\delta_{i}, \beta_{i}$ are $a_{i}$ and $b_{i-1}$ (with subscripts taken $\bmod (n+1)$ ), and such that $\delta_{i} \subset C$ and $\beta_{i} \subset \hat{h}(C)$. Consider the arc $\alpha_{0}$. There exists an arc $\eta_{1} \subset h\left(\alpha_{0}\right) \cap D$ which has one endpoint in $\delta_{0}$ and the other in $\delta_{1}$, and is "closest" to $p$, where by closest we mean that $\eta_{1}$ separates $p$ from all other such arcs of $\hat{h}\left(\alpha_{0}\right) \cap D$ in $D$. Inductively define $\eta_{i}$ as the arc in $h\left(\eta_{i-1}\right) \cap D$ with endpoints in $\delta_{0}$ and $\delta_{1}$, which is closest to $p$. Due to feature (6) above, $\eta_{i}$ is closer to $p$ than $\eta_{i-1}$. Let $U=\lim _{i>0}$ inf $\eta_{i}$. (See [CV] p. 253 for a definition of lim inf.) Since each $\eta_{i}$ contains a point from $\delta_{0}$ and $\delta_{1}$, the set $U$ is nonempty. By the Brown lemma, $p \in U$. Since each $\eta_{i}$ is compact and connected, it follows also that $U$ is compact and connected. Since $\eta_{i} \subset \hat{h}\left(\eta_{i-1}\right)$ we have that $\hat{h}^{-1}(U) \subset U$. 


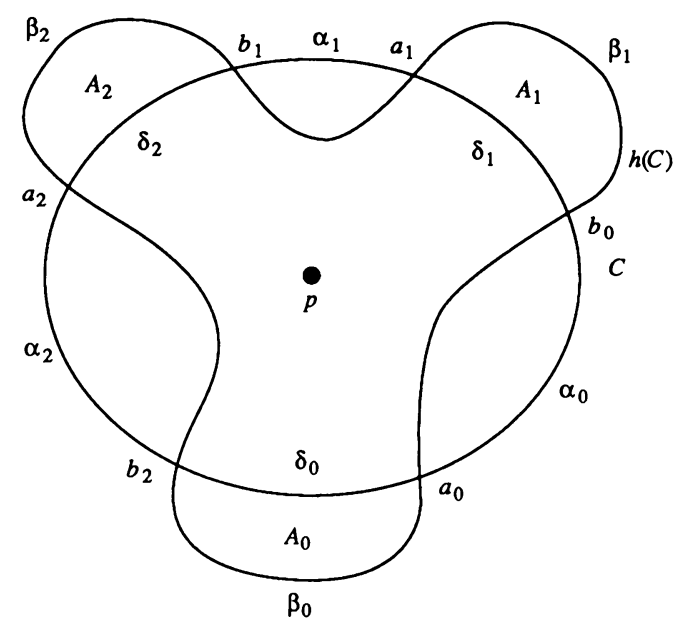

Figure 1.

Let $U_{0}$ be the irreducible (with respect to inclusion), compact, connected, $h^{-1}$ invariant subset of $U$ containing a point of $U \cap \delta_{0}$ and $p$. Such a set exists by the Brouwer reduction theorem. We claim that $U_{0} \cap C \subset \delta_{0}$. By construction $U_{0} \cap C \subset \delta_{0} \cup \delta_{1}$. Assume that $U_{0}$ meets $\delta_{1}$. In the same way the we constructed $U$ using $\hat{h}$ iterates starting with the arc at $\alpha_{0}$, construct the stable set $S_{0}$ from $\alpha_{0}$ to $p$ using $\hat{h}^{-1}$ iterates starting with the arc $\beta_{1}$. It follows that $\left(S_{0}-p\right) \cap U_{0} \neq \varnothing$. Let $x$ be a point in this intersection. Since $x \in S_{0}-p$, there exists an $N>0$ such that $\hat{h}^{-N}(x) \notin D$. However, by feature (6) above, $\hat{h}^{-n}(x) \notin D$ for all $n \geq N$. But, $x \in U_{0}$, a contradiction.

Again, by the Brown lemma, if $x \in U$ then $\hat{h}^{-m}(x) \rightarrow p$ as $m \rightarrow \infty$. Since each point $x \in U_{0}$ tends to $p$ under $\hat{h}^{-1}$ and the measure of $p$ is zero, the measure of $U_{0}$ is also zero.

Assume that $U_{0}$ separates the plane. Let $K$ be a bounded domain of the complement of $U_{0}$ which necessarily lies in $D$. Let $E \subset K$ be a small disc such that $\hat{h}^{-1}(E) \cap E=\varnothing$. By the corollary to the Brouwer lemma we arrive at a contradiction.

Assume $x \in U_{i} \cap U_{j}$, where $x \neq p$ and $i \neq j$. Then there exists $N>0$ such that $h^{n}(x) \in A_{i}$. However, as wás shown earlier, $U_{j}$ does not meet $A_{i}$. Note. In the case that $n=0$ the above procedure gives rise to $U_{0}$, as $U_{0}$ and $U_{1}$ each have points in $\delta_{0}=\delta_{1}$.

For each $i$ construct $U_{i}$, and then in a similar fashion construct the stable sets $S_{i}$. The homeomorphism $h$ has been modified on $D$ thus $U_{i}$, and $S_{i}$, as constructed, are not the desired sets. However, $h=\hat{h}$ on the neighborhood $N(p) \subset D$ noted in feature (2). The stable and unstable sets exist on an open 
subset of $N(p)$. Thus one can construct a disc $D$ in $N(p)$ containing $p$ which the above properties for $C=b d(D)$. The last property then follows by construction.

Question. The above construction used the irreducible connected invariant continuum to obtain the stable and unstable sets. One could enlarge this set to obtain maximal connected stable and unstable sets. However, it is not known whether every point, whose $\alpha$ or $\omega$ set is the fixed point, must lie in this unstable or stable set.

\section{REFERENCES}

[Ar] V. I. Arnol'd, Mathematical methods of classical mechanics, Springer-Verlag, New York, 1978.

[Br $\left.{ }_{1}\right] \mathrm{M}$. Brown, A new proof of Brouwer's lemma on translation arcs, Houston J. Math. 10 (1984), $35-41$.

[ $\left.\mathrm{Br}_{2}\right] \frac{-}{469}$, Homeomorphisms of two-dimensional manifolds, Houston J. Math. 11 (1985), 455469.

[CV] C. Christensen and W. Voxman, Aspects of topology, Marcel Dekker, New York, 1977.

[De] R. Devaney, An introduction to chaotic dynamical systems, Benjamin/Cummings, Menlo Park, CA, 1986.

[Di] T. Ding, Boundedness of solutions of Duffing's equation, J. Differential Equations 61 (1986), 178-207.

[Fa] A. Fathi, An orbit closing proof of Brouwer's lemma on translation arcs, Enseign. Math. 33 (1987), 315-322.

[Hi] K. Hiraide (communicated by R. Williams).

[Le] J. Lewowicz, Expansive homeomorphisms of surfaces, preprint.

[Mo] D. Montgomery, Measure preserving homeomorphisms at fixed points, Bull. Amer. Math. Soc. 51 (1945), 949-953.

[PS] P. Pelikan and E. E. Slaminka, $A$ bound for the fixed point index of area preserving homeomorphisms of two manifolds, Ergodic Theory Dynamical Systems 7 (1987), 463-479.

[S1] E. E. Slaminka, Removing index 0 fixed points for area preserving maps of two manifolds, Trans. Amer. Math. Soc. (submitted).

Department of Mathematics, Auburn University, Auburn University, Alabama 368495307 